\title{
eROSITA camera array on the SRG satellite
}

\author{
Norbert Meidinger ${ }^{*}$, Robert Andritschke, Konrad Dennerl, \\ Valentin Emberger, Tanja Eraerds $\odot$, Olaf Hälker, Gisela Hartner, \\ Daniel Pietschner, and Jonas Reiffers \\ Max-Planck-Institut für extraterrestrische Physik, Garching, Germany
}

\begin{abstract}
The Spectrum-Roentgen-Gamma satellite with the extended roentgen survey with an imaging telescope array (eROSITA) x-ray telescope as scientific payload was successfully launched on July 13, 2019 and deployed in a 6-month halo orbit around the second Lagrange point of the Sun-Earth system. The telescope comprises an array of seven mirror systems with seven focal plane cameras. The spectroscopic CCD cameras are a further development of the very successful EPIC-PN camera on the XMM-Newton satellite, which is still operating after more than 20 years in space. The key component of the camera is the detector, which matches the large field of view of 1 deg to permit an all-sky survey in the energy range from 0.2 to $8 \mathrm{keV}$ with state-of-the-art energy resolution. The image area of the $\mathrm{PN}$-junction charge coupled device comprises $384 \times 384$ pixels. The pixel size of $75 \times 75 \mu \mathrm{m}^{2}$ each matches the angular resolution of the mirror system. Readout of the full frame is achieved in $9.18 \mathrm{~ms}$ but for thermal and onboard event preprocessing reasons, the time resolution is slowed down to $50 \mathrm{~ms}$. The photon entrance window of five of the seven CCDs is equipped with an optical blocking filter, which proved to be advantageous. The improved concept and design of the eROSITA cameras will be explained as well as their operation and performance in space. (1) The Authors. Published by SPIE under a Creative Commons Attribution 4.0 Unported License. Distribution or reproduction of this work in whole or in part requires full attribution of the original publication, including its DOI. [DOI: 10.1117/1.JATIS.7.2.025004]
\end{abstract}

Keywords: eROSITA; focal plane camera; PNCCD; Spectrum-Roentgen-Gamma; x-ray detector; $\mathrm{x}$-ray telescope.

Paper 21018AS received Feb. 3, 2021; accepted for publication May 3, 2021; published online May 24, 2021.

\section{Introduction}

The extended roentgen survey with an imaging telescope array (eROSITA) $\mathrm{x}$-ray telescope has been developed to perform an all-sky survey in the energy band from 0.2 to $8 \mathrm{keV}$. It is the primary instrument of the Spectrum-Roentgen-Gamma mission, ${ }^{1}$ which was successfully launched on July 13, 2019, from the Baikonour cosmodrome.

After satellite launch and commissioning of the spacecraft, the eROSITA telescope cover was opened on July 23, 2019, starting the outgassing period of eROSITA. The detectors and optical blocking filters were not protected by a vacuum vessel but launched under ambient conditions. After a sufficiently long outgassing period, cool-down of the eROSITA detectors started on August 22, 2019, followed by camera commissioning. First light with just two telescope modules (TMs) took place on September 15, 2019, and finally with all seven TMs on October 16 to 18, 2019, using a region in the Large Magellanic Cloud (LMC) as observation target. When the calibration and performance verification program was finished, the all-sky survey started on December 13, 2019.

Eight complete scans are planned and each one takes 6 months. The all-sky survey program will be followed by a phase of pointed observations, expected to start in late 2023 or early 2024. The eROSITA design driving science is the detection of large samples of galaxy clusters up to redshifts $z>1$, in order to study the large-scale structure of the Universe and test cosmological models including dark energy. In addition, eROSITA is expected to yield a sample of a few

*Address all correspondence to Norbert Meidinger, nom@mpe.mpg.de 


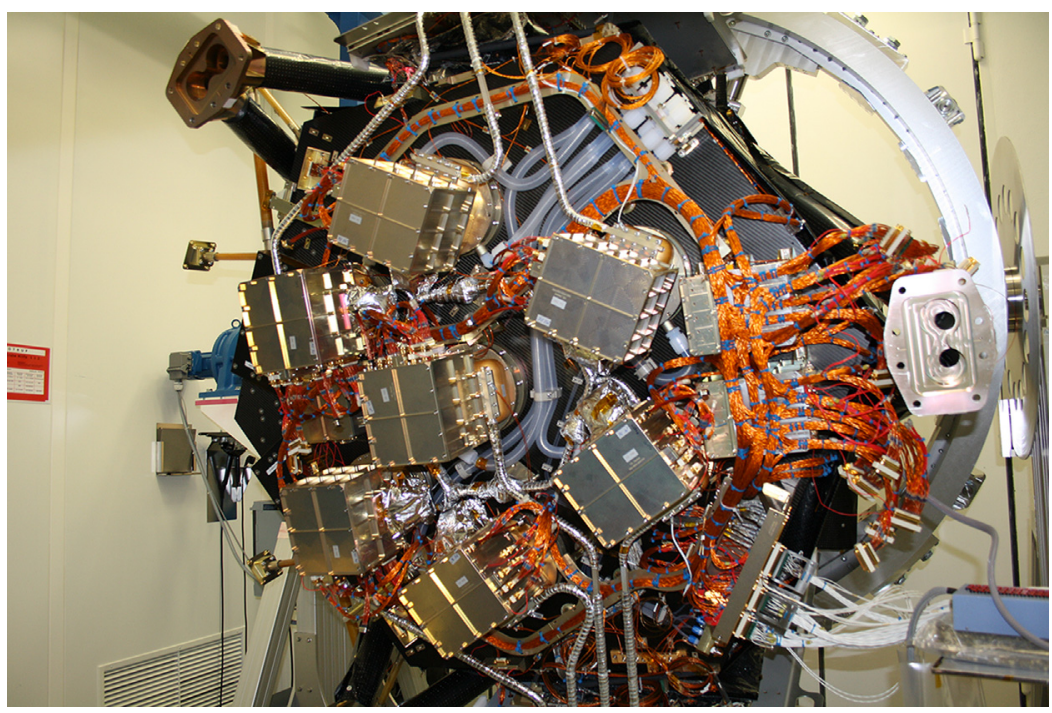

Fig. 1 eROSITA focal plane camera array (seen from the back) with one camera in the center and six cameras surrounding it (courtesy of $P$. Friedrich).

million AGN, including obscured objects, revolutionizing our view of the evolution of supermassive black holes. ${ }^{1}$

The eROSITA instrument comprises an array of seven identical and co-aligned telescopes. The instrument has a diameter of $\sim 1.9 \mathrm{~m}$ and a height of $3.2 \mathrm{~m}$ (in launch configuration). Its total weight is $808 \mathrm{~kg}$ and the maximum power consumption is $522 \mathrm{~W}$. A maximum data rate of $600 \mathrm{MB}$ per day is planned.

The key components of each telescope are the mirror system and the focal plane camera at a distance of $1600 \mathrm{~mm}$ (see Fig. 1). ${ }^{2}$ A mirror module comprises 54 nested nickel shells of Wolter-I type coated with gold. The half energy width is $\sim 16 \operatorname{arcsec}$ (on axis) at an energy of $1.5 \mathrm{keV} .^{1}$ Dedicated to the mirror is a spectroscopic focal plane camera with a field of view of $1.0 \times 1.0$ square deg. The focal plane is offset slightly from the best focus position such that the angular resolution is 18 arcsec on axis, but the average angular resolution over the field of view is improved.

Its sensor is an advanced version of the XMM-Newton PN-junction charge coupled device (PNCCD) device. ${ }^{3}$ It is also back-illuminated and the chip thickness of $450 \mu \mathrm{m}$ is fully depleted but equipped with a frame store. ${ }^{2}$ The image area of $28.8 \mathrm{~mm} \times 28.8 \mathrm{~mm}$ as well as the pixel size of $75 \times 75 \mu \mathrm{m}^{2}$ are matched to the field of view and angular resolution of the mirror system, respectively. The pixel size corresponds to a sky angle of $9.6 \operatorname{arcsec} \times 9.6 \operatorname{arcsec}$. Signal readout of a frame is accomplished within $9.18 \mathrm{~ms}$. Afterward, the analog signal processor, the so-called CAMEX (CMOS amplifier and multiplexer) ASIC, ${ }^{4}$ is switched into standby mode. Thus the heat dissipation of the readout ASIC is minimized, while the PNCCD continues to integrate x-ray signals. Extending the exposure time per frame to $50 \mathrm{~ms}$ means a minimization of the heat dissipation of the focal plane detector to only $0.7 \mathrm{~W}$. This means that the readout ASIC is switched 20 times per second into standby mode during nominal detector operation. By this method, the optimum temperature of $-95^{\circ} \mathrm{C}$ with respect to radiation damage mitigation ${ }^{5}$ should be achieved for the array of seven detectors according to the thermal design with two radiator panels and associated heat pipe system. Furthermore, the 50-ms time resolution provides sufficient time for onboard preprocessing of the CCD signals of a frame. The readout of the seven eROSITA detectors is not synchronized but free running. The time stamping of the events is synchronized to a $1-\mathrm{Hz}$ master clock that is common to the spacecraft. The data reduction is necessary because the daily telemetry rate from the spacecraft in the L2 halo orbit would not allow for transmission of all pixel signals to ground.

Back illumination of the ultra-thin entrance window and full depletion of the 450- $\mu \mathrm{m}$ sensor thickness lead to a high quantum efficiency (QE) for both, low and high x-ray energies. Actually, 
Table 1 Characteristics of the eROSITA detector.

\begin{tabular}{|c|c|}
\hline Sensor & PNCCD \\
\hline Illumination type & Back illumination \\
\hline \multirow[t]{2}{*}{ Image area } & $384 \times 384$ pixels \\
\hline & $28.8 \mathrm{~mm} \times 28.8 \mathrm{~mm}$ \\
\hline Pixel size (image area) & $75 \mu \mathrm{m} \times 75 \mu \mathrm{m}$ \\
\hline Pixel size (frame store area) & $75 \mu \mathrm{m} \times 51 \mu \mathrm{m}$ \\
\hline Readout ASIC & $\begin{array}{l}\text { 128-channel eROSITA CAMEX } \\
\text { (3 ASICs per PNCCD) }\end{array}$ \\
\hline Read noise & 2.5 electrons ENC rms \\
\hline \multirow[t]{2}{*}{ Energy resolution } & $\operatorname{FWHM}(0.53 \mathrm{keV}) \approx 62 \mathrm{eV}$ \\
\hline & $\operatorname{FWHM}(5.9 \mathrm{keV}) \approx 140 \mathrm{eV}$ \\
\hline Nominal operating temperature & $-95^{\circ} \mathrm{C}$ (best with respect to radiation damage) \\
\hline \multirow{5}{*}{$\begin{array}{l}\text { QE (calculated) without/with on-chip filter } \\
\text { (note: the filter in filter wheel is not considered here) }\end{array}$} & $E=0.3 \mathrm{keV}: 66 \% / 16 \%$ \\
\hline & $E=0.5 \mathrm{keV}: 80 \% / 53 \%$ \\
\hline & $E=1.0 \mathrm{keV}: 95 \% / 89 \%$ \\
\hline & $E=5.0 \mathrm{keV}: 99.7 \% / 98.7 \%$ \\
\hline & $E=10 \mathrm{keV}: 98.2 \% / 98.1 \%$ \\
\hline Readout time & $9.18 \mathrm{~ms}$ \\
\hline Transfer time to frame store & $115 \mu \mathrm{s}$ \\
\hline Time resolution & $50 \mathrm{~ms}$ \\
\hline
\end{tabular}

the QE for low X-ray energies is determined by the filter that is needed for attenuation of optical light since the PNCCD is also sensitive to visual light. Two different methods of light blocking (by at least five orders of magnitude) were applied for the seven cameras of the eROSITA instrument.

a. The direct deposition of a 200-nm-thick Al layer on the photon entrance window of the sensor chip. The filter wheel is in this case equipped with a 200 -nm-thick polyimide layer. Such a warm filter is advantageous to prevent contamination on the cold CCD chip. The deposition of on-chip filters is a new method and the light blocking factor per thickness is smaller than that of external filters, which are based on a mature technology.

b. The conventional way to use an external filter mounted in the filter wheel, which is accommodated between the mirror system and the detector. The external filter consists of $100 \mathrm{~nm}$ of $\mathrm{Al}$ deposited on a 200-nm-thick polyimide substrate.

Both external filters, i.e., with and without aluminum coating, are self-supporting without use of a mesh.

The camera assembly consists of the following subsystems: camera head, camera electronics (CE), and filter wheel. The camera head comprises the detector, the detector housing, the proton shield, and a heat pipe for cooling of the detector. The supply, control, and data acquisition electronics for the detector operation is contained in the CE, which is accommodated on the back side of the camera head. In front of the camera head, the filter wheel is mounted.

The configuration of camera head, CE, and filter wheel is shown in Fig. 2. 


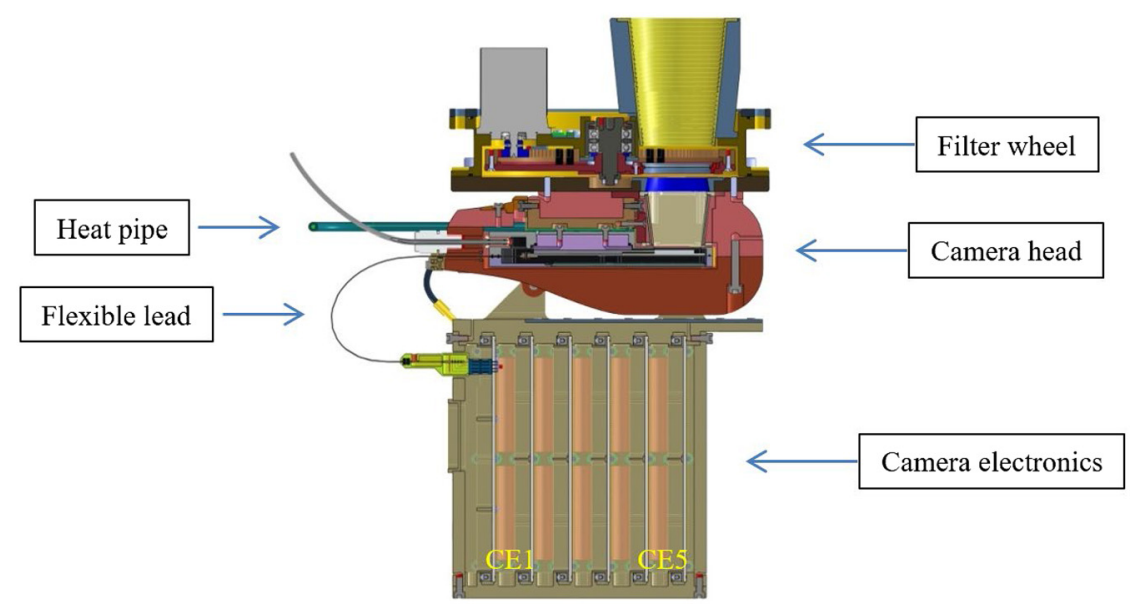

Fig. 2 Sectional view of camera assembly with filter wheel on top, camera head in the center and $\mathrm{CE}$ at the bottom. The detector is connected in a minimum distance to the frontend PCB by a flexible lead with connector (on left-hand side). The heat pipe links the detector to the radiator for cooling. The height of the camera assembly is $378 \mathrm{~mm}$.

\section{Detector}

The detector consists of the following main components: the PNCCD chip, three CAMEX analog signal processor ASICs for readout, the detector board, and the detector housing, which provides the mechanical and thermal interfaces.

\subsection{PNCCD Detector}

The key component of each eROSITA CCD detector is the PNCCD sensor chip. It is based on the development done for the EPIC-PN camera on XMM-Newton, ${ }^{3}$ but several significant improvements have been implemented (Table 1):

- Frame transfer $C C D$. A frame store was added to the image area to minimize the percentage of out-of-time events, which smear the source photons over the charge transfer channel (see Fig. 3). This effect causes readout streaks when bright point sources are observed. With a transfer time of the image into the shielded frame store section of $115 \mu \mathrm{s}$, the out-of-time fraction could be reduced to $0.23 \%$. For comparison, the EPIC-PN detector on XMM-Newton shows in full frame mode an out-of-time fraction of $6.3 \%$.

- Charge transfer efficiency (CTE). A CTE improvement was achieved by an optimized design and fabrication process of the CCD charge transfer channel. The eROSITA CTE value of about 0.99998 has to be compared to the EPIC-PN detector CTE of 0.9995, measured under comparable conditions. This means a substantial reduction of charge transfer loss per pixel by about a factor of 25 compared to the PNCCD applied on XMM-Newton.

- The front side of the CCD, which is covered with the transfer gates and transistors, has been passivated to prevent the risk of shorts caused by conductive particles. This is a lesson learned from the EPIC-PN detector development.

- An optical blocking filter made of aluminum was deposited on the photon entrance window of the CCD. The CCDs for TM1, TM2, TM3, TM4, and TM6 are equipped with the 200-nm-thick on-chip filter, while it was decided to use no on-chip filter for the CCDs for TM5 and TM7 as done for EPIC-PN. The advantage of an on-chip filter is the minimum distance between filter and sensor as well as the robustness against acoustic noise and vibrations during satellite launch. The disadvantage is the slightly higher light transmission per aluminum layer thickness, which is caused by constraints in the deposition on a complex sensor chip. The sputtered ultra-thin metal layer forms grains and their size depends on several processing parameters. Voids between grains increase the transmission of light. ${ }^{6}$ 


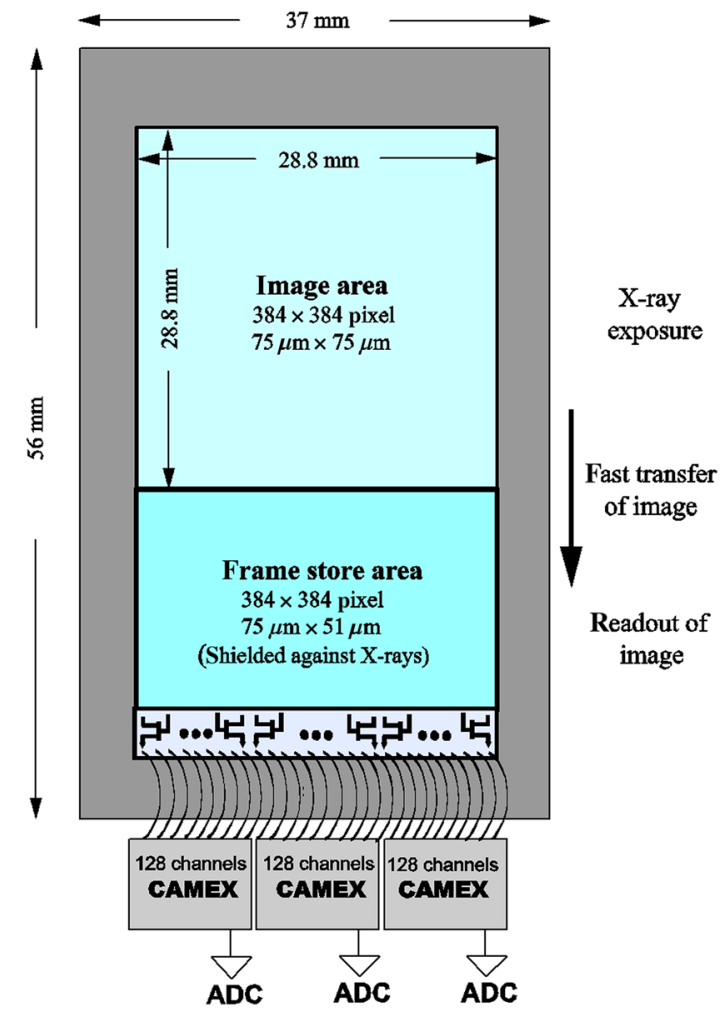

Fig. 3 Architecture and dimensions of an eROSITA PNCCD detector. After the integration time $(50 \mathrm{~ms})$ is finished, the image is transferred within $0.115 \mathrm{~ms}$ to the adjacent frame store section of the CCD. The 384 CCD channels are read out simultaneously by three 128-channel VLSI CAMEX chips. During signal processing of the next row, the signals of the previous row are serialized to the CAMEX output buffer, which feeds the analog signals into an ADC for digitization. The readout of an image takes $9.18 \mathrm{~ms}$.

- A read noise of 2.5 electrons equivalent noise charge (ENC) rms was achieved for the eROSITA detector in the flight camera assembly. The lower noise compared to the EPIC-PN detector developed for XMM-Newton (by about a factor of two) was accomplished by virtue of: first, a custom $n$-channel metal oxide semiconductor (MOS) transistor for charge clear on the anode after readout and second, a design optimization of the eROSITA CAMEX ASIC. The lower read noise allows for a lower event threshold and thus more complete recombination of split event signals, leading to a more accurate determination of the photon energy.

- The spectral response at low energies was substantially improved for the eROSITA CCDs. Although, for example, the carbon line at $277 \mathrm{eV}$ shows no peak in the measurements with the EPIC-PN CCD detector on XMM-Newton, the eROSITA detector yields a Gaussian-like line spectrum (see Sec. 4.3). The difference is caused by a reduction of partial event generation due to a change to (100) silicon wafers, improvement of the photon entrance window doping profile, and a more complete charge collection in case of the eROSITA CCDs.

The eROSITA PNCCDs were produced on wafers with $150-\mathrm{mm}$ diameter and $450-\mu \mathrm{m}$ thickness. The entire thickness of the back-illuminated CCD is fully depleted and thus sensitive to $\mathrm{x}$-rays. The majority of the detected events are split with signal charge spreading over up to four pixels. This is different from the EPIC-PN CCD, which had a larger pixel size of $150 \mu \mathrm{m} \times$ $150 \mu \mathrm{m}$ and therefore signal collection occurred mainly in one pixel.

The eROSITA PNCCD development and production was done at the Max-Planck-Institut Halbleiterlabor, which was operated at that time by the Max-Planck-Institut für extraterrestrische Physik (MPE) and the Max-Planck-Institut für Physik. The CCD development was done in 


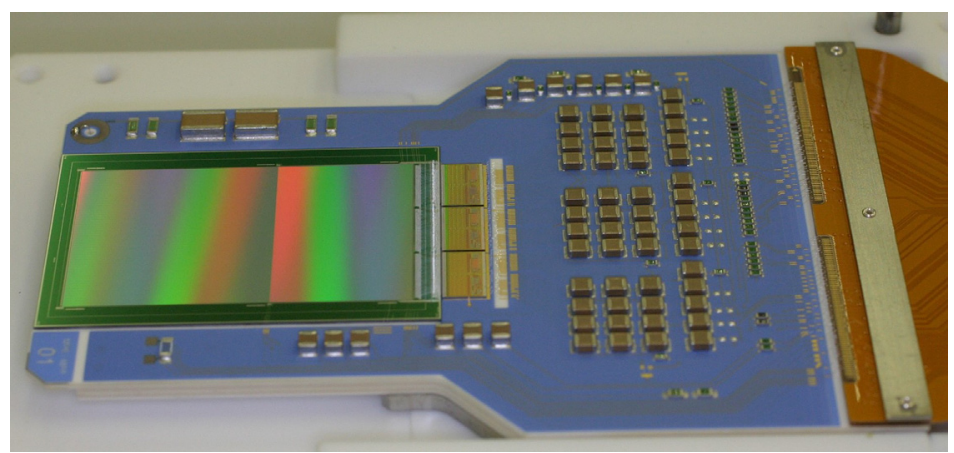

Fig. 4 eROSITA CCD detector consisting of the detector board (blue color): eROSITA PNCCD (left), three CAMEX ASICs (center), and rigid flex printed board as electrical interface (right).

cooperation with the company PNSensor. The produced PNCCDs were pretested by a coldchuck probe station of MPE, which allowed full operation on die-level, to select the best sensor chips for integration into the flight detector modules.

The sensor chip and the three CAMEX ${ }^{4}$ ASICs are mounted on a multi-layer detector board produced in thick film technology at the Max Planck Institute (MPI) for physics. It is connected by a flexible lead or more precisely, a rigid flex printed board with a 152-pin connector as electrical interface, to the front-end printed circuit board (PCB) of the CE (see Figs. 2 and 4). Before integration of the CCD sensors, the detector module was fully assembled and the function of the eROSITA CAMEX analog signal processor chips was tested. Three of these 128-channel ASICs are necessary per PNCCD for the readout of the 384 fully column-parallel CCD-channels as shown in Fig. 4. Each channel is terminated by an anode, which is connected to the gate of a monolithically integrated $n$-channel JFET for on-chip signal amplification. A wire bond connects the source of the JFET to a dedicated channel of CAMEX. A current source at the input of the CAMEX channel biases the JFET to operate it in source follower mode.

The signal charge transferred to the anode is automatically cleared by a "weak" avalanche breakdown mechanism which occurs between gate and drain in the transistor channel. ${ }^{7}$ It is self-regulating depending on the current to the anode. Since protons in space can temporarily generate large amounts of charges in the pixels, a second externally controllable clear mechanism was implemented in the eROSITA PNCCDs. By means of an on-chip transistor of MOS type, the charge on the anodes can be cleared during the integration time without any increase of read noise. Further details on the development of the eROSITA PNCCD detector can be found in Refs. 5, 8-10.

\subsection{Detector Housing, Thermal Interface, and Graded-Z Shielding}

The CCD detector is mounted into an aluminum housing, which is thermally decoupled by blades from the surrounding warm proton shield made of copper. This is necessary for cooling the detector to a temperature more than $100^{\circ} \mathrm{C}$ lower than the mechanical structure of the camera assembly. The thermal interface of the detector is mounted to the detector housing (see Fig. 5) and linked via heat pipes to two camera radiator panels. The reliable principle of passive cooling is used for the eROSITA cameras.

For minimization of the instrumental background, a graded $Z$ shield $(Z=$ atomic number) was implemented around the PNCCD sensor chip. The sensor side opposite to the mirror system is shielded by a beryllium plate, which is mounted inside the aluminum housing. The sensor side facing the mirror is equipped with a boroncarbide plate, which shields the frame store area of the PNCCD. The beryllium and the boroncarbide plates are enclosed by the aluminum housing of the detector and this again is enclosed by the copper proton shield. The aperture of the camera is also equipped with such a graded- $Z$ shielding. The basic idea of the graded- $Z$ shielding is that fluorescence radiation generated by particles in space in a layer will be absorbed in the next inner layer and due to the lower atomic number of the inner layer, the fluorescence yield and energy become lower. It is obvious that the effect of the shield reaches its limit due to the fact that (i) all used materials have impurities, (ii) the shield inside the camera cannot cover the CCD entirely, 


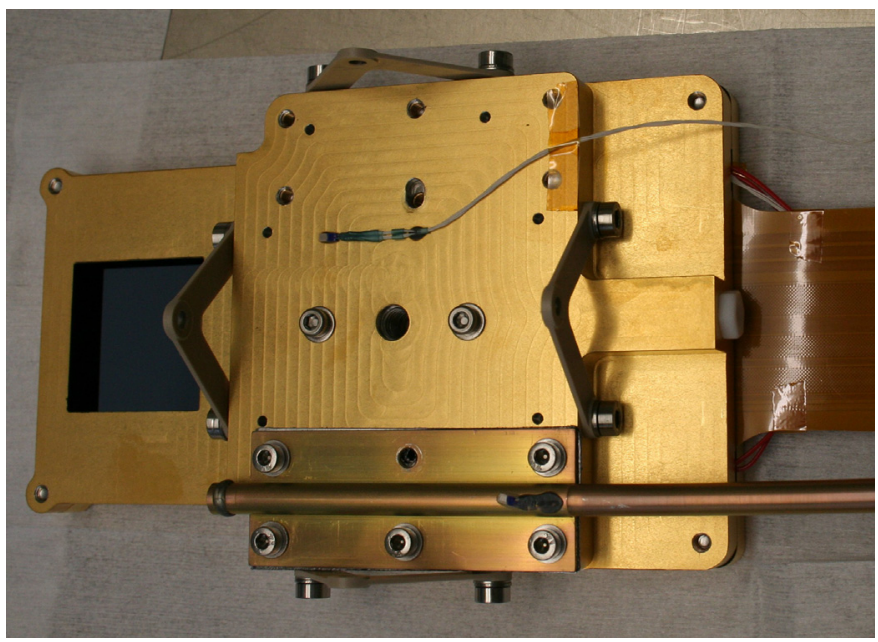

Fig. 5 CCD module mounted in its housing. The aperture to illuminate the image area of the CCD is shown on the left-hand side while on the right-hand side the rigid flex printed board (electrical interface) and underneath the constant conductance heat pipe (thermal interface) are visible.

and (iii) that the image area of the detector faces parts of the instrument which are not equipped with a graded- $Z$ shield.

The copper shield has a thickness of $\sim 3 \mathrm{~cm}$ and serves as proton shield. Thereby, the CCD as well as the CAMEX ASICs are sufficiently protected against the non-ionizing energy deposition of protons, which generates lattice defects in the silicon device. Defects can act as electron traps in the depth of the charge transfer and degrade the CTE. A loss of signal electrons, even if it is corrected on the average, causes a degradation of energy resolution.

\section{Camera Assembly with Filter Wheel and Electronics}

\subsection{Filter Wheel}

The filter wheel, shown in Fig. 2, is mounted in front of the camera toward the mirror system. It provides four different positions, which are controlled by a stepper motor and the position sensing is accomplished by Hall sensors and magnets (see Fig. 6).

a. Filter position to attenuate optical and UV light, which would generate electrons in the $\mathrm{CCD}$. These electrons would interfere with the signal electrons from the $\mathrm{x}$-ray photons and corrupt the signal energy.

Since camera TM5 and TM7 are not equipped with an on-chip light blocking filter, their associated filters in the filter wheel are equipped with a 100-nm-thick Al layer on a 200-nmthick polyimide substrate. The other five cameras TM1 to TM4 and TM6 have only a 200-nm thick polyimide foil mounted in the filter wheel. This position is used as standard observation mode. The polyimide layer serves also to minimize contamination because the external filter is kept at warm temperature but the on-chip filter has the low temperature of the CCD chip. The TMs with on-chip optical blocking filter permit the option to observe without the polyimide filter and thus higher QE in case contamination is no issue.

b. Calibration source position with an ${ }^{55} \mathrm{Fe}$ radioactive source, which generates in addition fluorescence photons of the target materials $\mathrm{Al}$ und $\mathrm{Ti}$ (see Fig. 7). The source provides thereby three main spectral lines at $5.9(\mathrm{Mn}-\mathrm{K} \alpha), 4.5(\mathrm{Ti}-\mathrm{K} \alpha)$, and $1.5 \mathrm{keV}(\mathrm{Al}-\mathrm{K})$.

c. Closed position for protection and instrumental background measurements in space.

d. Open position for optimum outgassing. It allows optionally observations with higher QE in case of TM1 to TM4 and TM6, which are equipped with on-chip optical blocking filter. 


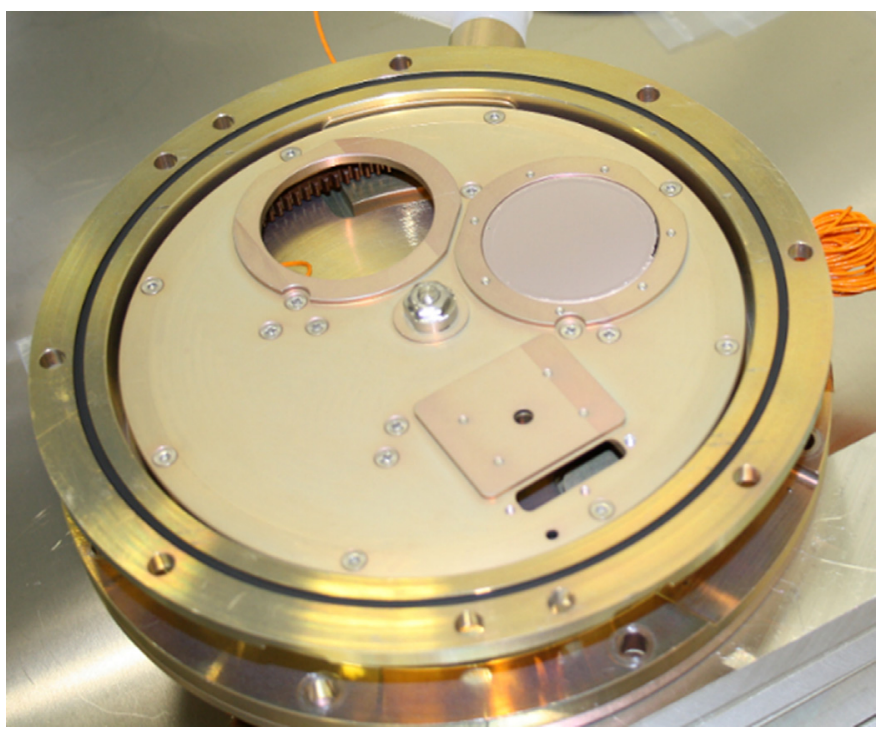

Fig. 6 eROSITA filter wheel with the four positions (clockwise from the top right): filter, calibration source, closed, and open position.

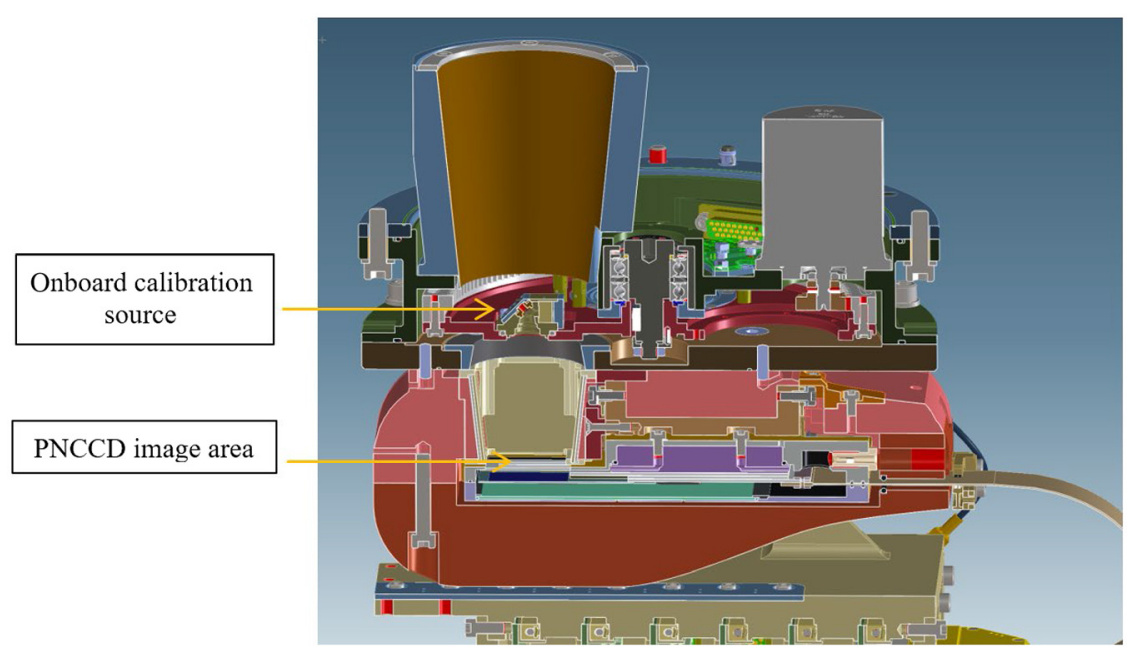

Fig. 7 Cross section of camera head and filter wheel in onboard calibration source position. In spite of the small distance between source and sensor chip of $63 \mathrm{~mm}$, the entire image area can be illuminated with the $\mathrm{x}$-ray photons of the onboard calibration source.

\subsection{Camera Electronics}

The two main groups of electronic systems for the operation of the seven cameras are the interface and thermal controller (ITC) that exists as nominal and redundant unit to avoid a single point of failure risk and the seven CE dedicated to the seven cameras. ${ }^{11}$

\subsubsection{Interface and thermal controller}

The ITC is the electrical and logical interface between the spacecraft and the eROSITA telescope. It receives the power from the spacecraft (via electronic latch relay) and distributes it to the eROSITA subsystems. Commands are received via MIL-STD-1553 serial interface from the spacecraft controller and transmitted to the seven CE. Furthermore, the ITC receives a $1-\mathrm{Hz}$ time signal from the spacecraft and distributes it to the seven eROSITA cameras. 
The ITC receives in return the data from the seven CE for processing and storage in the mass memory. The data stored in the mass memory are sent on request to the radiocomplex of the spacecraft for downlink telemetry to the ground stations.

Another task of the ITC apart from the control of the mirror temperatures is the control of the temperature of the CCD detector by heating the reservoirs of the variable conductance heat pipes. ${ }^{9}$ The key component of the ITC is a Xilinx Virtex-4 FPGA (field programmable gate array) with two embedded Power-PC cores.

\subsubsection{Camera electronics}

The CE supplies the voltages and signals for the operation of the CCD detector. Apart from the filter wheel, it controls the readout of the detector, i.e., of the PNCCD sensor and CAMEX ASICs, with three ADCs and a sequencer. Another main task of the CE is the on-board signal processing of the CCD frames. This includes correction of offset and common mode and enables event filtering by use of a pixel dependent event threshold for the purpose of data reduction. Signals exceeding an upper energy threshold are caused by cosmic ray particles, high-energy x-rays, or their secondaries. These signals are discarded but their occurrence is registered. The science and housekeeping data are transmitted to the ITC. From the ITC, the CE receives in return commands and a time signal. All seven CE are powered by the ITC. Key component of the CE is the same type of Xilinx Virtex-4 FPGA as used for the ITC.

The CE comprises five boards named CE1 to CE5, which provide the following functions: CE1 is the front-end board and directly connected to the CCD detector (see Fig. 2). It buffers the analog detector signals, generates the CCD clock pulses, and distributes the signals between detector and CE. CE2 is a voltage control board that generates the voltages for operation of the CCD and its readout ASICs. In addition, analog housekeeping data (currents, voltages, temperatures, etc.) are digitized and analog voltages are generated according to telecommands. CE3 generates and supplies the necessary voltage of about $250 \mathrm{~V}$ for full depletion of the CCD sensor and the floating voltages for the clock pulse drivers. Furthermore, it controls the filter wheel subsystem. CE4 is responsible for telecommanding, telemetry, and onboard event processing. The main components of this duplex board are three 14-bit ADCs for digitization of the detector signals (one ADC per CAMEX) and the FPGA unit, which provides also the sequencer function. The radiation-hard Virtex-4 FPGA enables the digital logic of the system including the filter wheel. By use of triple module redundancy (TMR) design technique, single-event upsets (SEUs) can be prevented. CE5 contains the main voltage converters, which are supplied with $+27 \mathrm{~V}$ but insulated from the spacecraft. It is realized in cold redundant version to avoid a single point of failure risk for a focal plane camera assembly.

\section{4 eROSITA Camera Performance}

\subsection{Performance Tests at MPE}

All CCD detector modules were tested after assembly in the small GEPARD test facility at MPE, which is equipped with an ${ }^{55} \mathrm{Fe}$ radioactive source. There, the commissioning of the detector after assembly was performed, the operating voltages optimized and its performance characterized.

After the test in the GEPARD facility, the detector was integrated in its housing and subsequently in the camera head. Further performance tests and calibration were done with the complete camera assembly in the larger PUMA x-ray test facility at MPE. The setup in PUMA permitted exposure of the detector by the onboard calibration source of the camera assembly as well as by a multi-target x-ray tube mounted at a distance of several meters from the camera.

The complete camera array, i.e., the operation of all seven cameras simultaneously, was tested in the so-called end-to-end test of the eROSITA instrument. This test was performed in the large PANTER test facility of MPE. 
Table 2 Radiation damage effect on the average energy resolution of the entire CCD expressed as FWHM of the spectral line for three different operating temperatures at a 5.6-MeV proton fluence of $2.3 \times 10^{8}$ and $4.0 \times 10^{8} \mathrm{~cm}^{-2}$, which corresponds approximately to a 7.5 -year fluence with $\mathrm{CL}=90 \%$ and $\mathrm{CL}=95 \%$, respectively. A duration of 7.5 years has been planned as mission lifetime with 4 years dedicated to the all-sky survey and 3.5 years for selected observations and programs afterward. Even at a temperature of $-80^{\circ} \mathrm{C}$, no increase in read noise due to a rise of dark current was observed in the measurements. ${ }^{12}$

\begin{tabular}{|c|c|c|}
\hline Temperature $\left({ }^{\circ} \mathrm{C}\right)$ & FWHM $(277$ eV) & FWHM (5.9 keV) \\
\hline \multicolumn{3}{|c|}{$\Phi\left(5.6 \mathrm{MeV}\right.$-proton $\left./ \mathrm{cm}^{2}\right)=0$, corresponding to status without radiation damage } \\
\hline-80 & $52 \mathrm{eV}$ & $148 \mathrm{eV}$ \\
\hline-90 & $52 \mathrm{eV}$ & $148 \mathrm{eV}$ \\
\hline-100 & $51 \mathrm{eV}$ & $146 \mathrm{eV}$ \\
\hline \multicolumn{3}{|c|}{$\Phi\left(5.6 \mathrm{MeV}\right.$-proton $\left./ \mathrm{cm}^{2}\right)=2.3 \times 10^{8}$, corresponding to about 7.5 years mission lifetime with $\mathrm{CL}=90 \%$} \\
\hline-80 & $55 \mathrm{eV}$ & $181 \mathrm{eV}$ \\
\hline-90 & $54 \mathrm{eV}$ & $168 \mathrm{eV}$ \\
\hline-100 & $55 \mathrm{eV}$ & $158 \mathrm{eV}$ \\
\hline \multicolumn{3}{|c|}{$\Phi\left(5.6 \mathrm{MeV}\right.$-proton $\left./ \mathrm{cm}^{2}\right)=4.0 \times 10^{8}$, corresponding to 7.5 years mission lifetime with $\mathrm{CL}=95 \%$} \\
\hline-80 & $57 \mathrm{eV}$ & $215 \mathrm{eV}$ \\
\hline-90 & $56 \mathrm{eV}$ & $186 \mathrm{eV}$ \\
\hline-100 & $60 \mathrm{eV}$ & $166 \mathrm{eV}$ \\
\hline
\end{tabular}

\subsection{Radiation Damage}

Long-term stability of performance is essential for the success of space projects. Therefore, the potential radiation damage to the CCD had been accurately evaluated. Most critical is the nonionizing energy loss of particles in the PNCCD. It generates point defects in the silicon lattice. They act as traps for signal electrons and ultimately cause a gradual degradation of the energy resolution of the detector over the course of the mission.

Considering cosmic protons and solar protons at the L2 halo orbit, we obtain behind the shielding a total $5.6 \mathrm{MeV}$ proton equivalent fluence of about $2.4 \times 10^{8} \mathrm{~cm}^{-2}$ at the focal plane during a mission lifetime of 7.5 years at a confidence level (CL) of $90 \%$ (see Ref. 9). Generation of neutron secondaries in the copper proton shield was taken into account in the analysis.

Raising the CL for solar protons to $95 \%$ yields a total fluence of about $4.0 \times 10^{8} \mathrm{~cm}^{-2}$, with protons and secondary neutrons contributing $55 \%$ and $45 \%$, respectively.

We tested the radiation damage of the detector with protons at the TANDEM accelerator of the Maier-Leibnitz-Laboratorium in Garching, Germany. For this purpose, CCD chips from the same batch as the flight devices were used and operated with prototype electronics. No proton shield and filter wheel were used in the setup. The degradation of the energy resolution was measured afterward for two different x-ray energies of $277 \mathrm{eV}$ and $5.9 \mathrm{keV}$ and as function of temperature (see Ref. 12). The results are summarized in Table 2.

\subsection{Spectral Performance}

Accurate spectral performance tests of a PNCCD detector of eROSITA type were carried out at the BESSY II synchrotron in Berlin in cooperation with the Physikalisch Technische Bundesanstalt. ${ }^{6}$ The complete camera assemblies in flight configuration consisting of the camera with detector, the CE, and the filter wheel were tested in the PUMA test facility. 


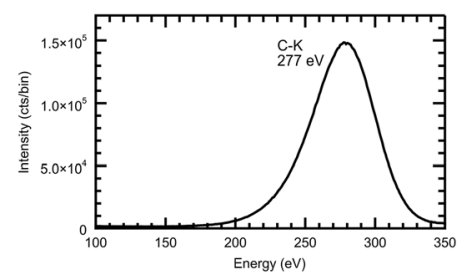

(a)

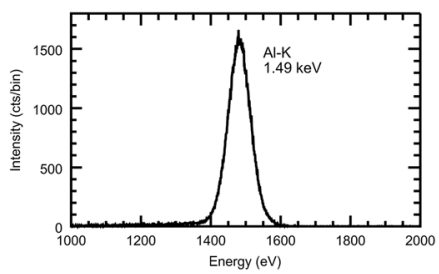

(b)

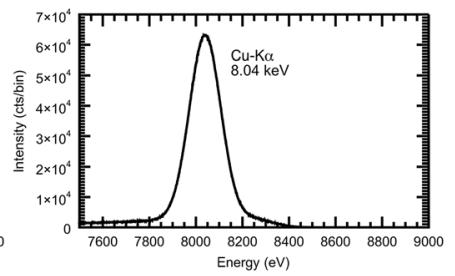

(c)

Fig. 8 With camera assembly TM5 measured spectra: (a) C-K spectrum with line energy of 277 eV and full-width at half-maximum (FWHM) of $50 \mathrm{eV}$ after accurate calibration (see Table 4); (b) Al-K spectrum with line energy of $1.49 \mathrm{keV}$ and FWHM of $75 \mathrm{eV}$; and (c) Cu- $\mathrm{K}_{\alpha}$ spectrum with line energy of $8.04 \mathrm{keV}$ and FWHM of $155 \mathrm{eV}$. The spectra show in addition to the fluorescence lines a Bremsstrahlung continuum, which is caused by the $\mathrm{x}$-ray tube.

The operating mode for the detector was chosen as used for space, e.g., switching the CAMEX readout ASIC 20 times per second into standby mode and back to normal operation.

The qualification model of the eROSITA cameras was extensively tested to validate the entire camera design. Apart from vibration and thermal cycling tests, in particular, the spectroscopic performance was studied in detail. Although the best CCDs were used for the flight cameras after a preselection process, the performance was extensively studied before the flight camera assembling process was started. The evaluation of the read noise of the system yielded an excellent value of about 2.5 electrons ENC rms. Spectra of three selected lines, C-K at $277 \mathrm{eV}, \mathrm{Al}-\mathrm{K}$ at $1.49 \mathrm{keV}$, and $\mathrm{Cu}-\mathrm{K}_{\alpha}$ at $8.04 \mathrm{keV}$, generated by the PUMA x-ray tube and measured with the TM5 eROSITA flight camera are shown in Fig. 8.

The energy resolution of the flight cameras after accurate calibration is presented in Sec. 4.5. The onboard calibration source for eROSITA is configured as an ${ }^{55} \mathrm{Fe}$ radioactive source mounted in the filter wheel, which illuminates a target made of titanium and aluminum. As a result, the $\mathrm{Mn}-\mathrm{K}_{\alpha}, \mathrm{Mn}-\mathrm{K}_{\beta}$ lines and $\mathrm{Al}-\mathrm{K}, \mathrm{Ti}-\mathrm{K}_{\alpha}, \mathrm{Ti}-\mathrm{K}_{\beta}$ fluorescence lines, the associated $\mathrm{Si}-\mathrm{K}$ escape peaks, and resulting pile-up combinations of these characteristic lines appear in the spectrum as shown in Fig. 9. The total photon flux amounts to 200 photons/frame, which decreases with time according to half-life of 2.737 years. Although the photon distribution is not uniform over the CCD image area because of the short distance between the compact

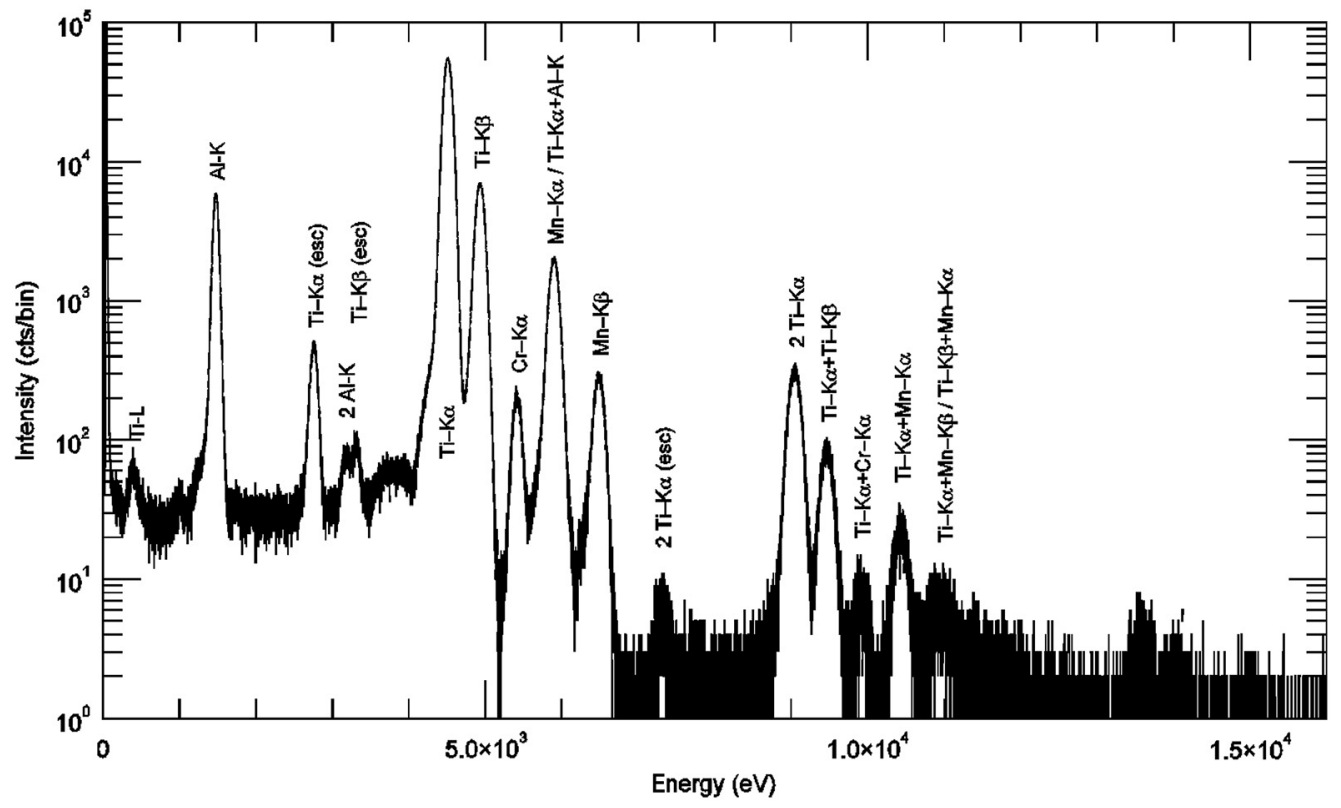

Fig. 9 Spectrum of the eROSITA onboard calibration source measured with an eROSITA camera. 


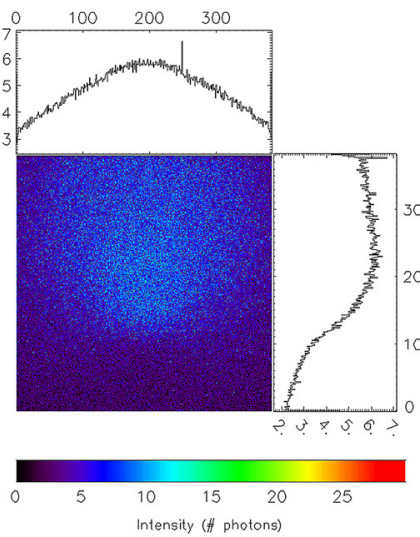

(a)

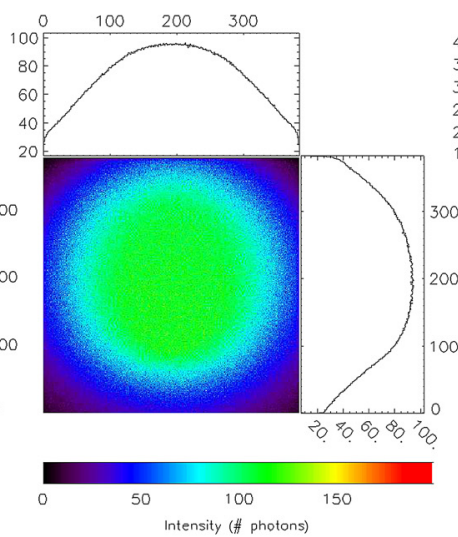

(b)

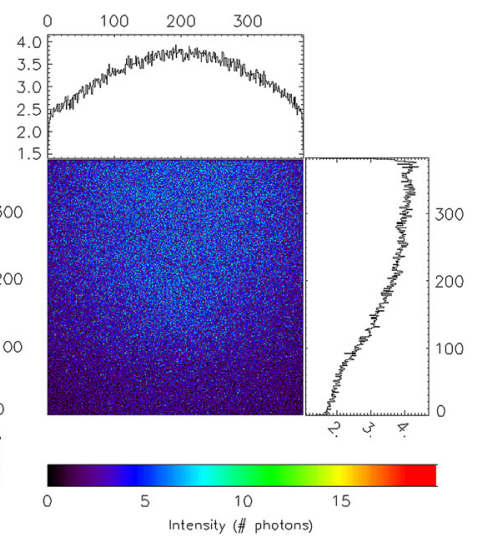

(c)

Fig. 10 Spatial distribution of the onboard calibration source photon intensity over the image area of the PNCCD. The intensity distribution of (a) the Al-K photons; (b) the $\mathrm{Ti}^{-\mathrm{K}_{\alpha, \beta}}$ photons; and (c) $\mathrm{Mn}-\mathrm{K}_{\alpha, \beta}$ photons.

calibration source and the CCD (see Fig. 7), the entire photon entrance window is illuminated by each of the three main spectral lines: $\mathrm{Al}, \mathrm{Ti}-\mathrm{K}_{\alpha, \beta}$, and $\mathrm{Mn}-\mathrm{K}_{\alpha, \beta}$ (see Fig. 10). Thus all CCD channels can be re-calibrated in space if changes occur, in particular a degradation of the CTE due to radiation damage or an alteration of the channel gains caused by a temperature change.

\subsection{Quantum Efficiency of X-Rays and Attenuation of Optical and UV Light}

Table 3 shows the QE of the seven camera assemblies TM1 to TM7 grouped in two categories: TM1 to TM4 and TM6 are equipped with on-chip filter and have a polyimide filter in the filter wheel. TM5 and TM7 have the optical blocking filter in the filter wheel and just thin siliconoxide and silicon-nitride layers on the silicon sensor. The listed QE values are based on measurements at the synchrotron radiation facility BESSY II combined with calculations. ${ }^{6}$ The error bars are on the order of $\leq 5 \%$. Higher QE can be achieved for TM1 to TM4 and TM6 at low energies by observing through the open position of the filter wheel if the accumulation of contamination turns out to be insignificant without a warm filter in front of the cold detector.

The purpose of the on-chip filter is the attenuation of optical and UV light. These values have been measured and are shown in Fig. 11. Further details can be found in Ref. 6. Blocking of optical and UV photons is necessary to avoid the interference of their signal electrons with those generated by x-ray photons, which would shift the energy and increase the noise.

Table 3 QE of camera assemblies including the filter in the filter wheel.

\begin{tabular}{lcc}
\hline \hline Spectral line energy & QE TM1 to TM4, TM6 (\%) & QE TM5, TM7 (\%) \\
\hline C-K: $277 \mathrm{eV}$ & 12.4 & 31.3 \\
O-K: $525 \mathrm{eV}$ & 42.2 & 51.3 \\
Cu-L: $930 \mathrm{eV}$ & 80.0 & 83.2 \\
Al-K: $1.49 \mathrm{keV}$ & 94.0 & 94.8 \\
Ti-K $\alpha: 4.51 \mathrm{keV}$ & 97.9 & 98.2 \\
Fe-K $\alpha: 6.40 \mathrm{keV}$ & 98.9 & 99.1 \\
Cu-K $\alpha: 8.04 \mathrm{keV}$ & 99.3 & 99.4 \\
Ge-K $\alpha: 9.89 \mathrm{keV}$ & 96.9 & 96.9 \\
\hline \hline
\end{tabular}




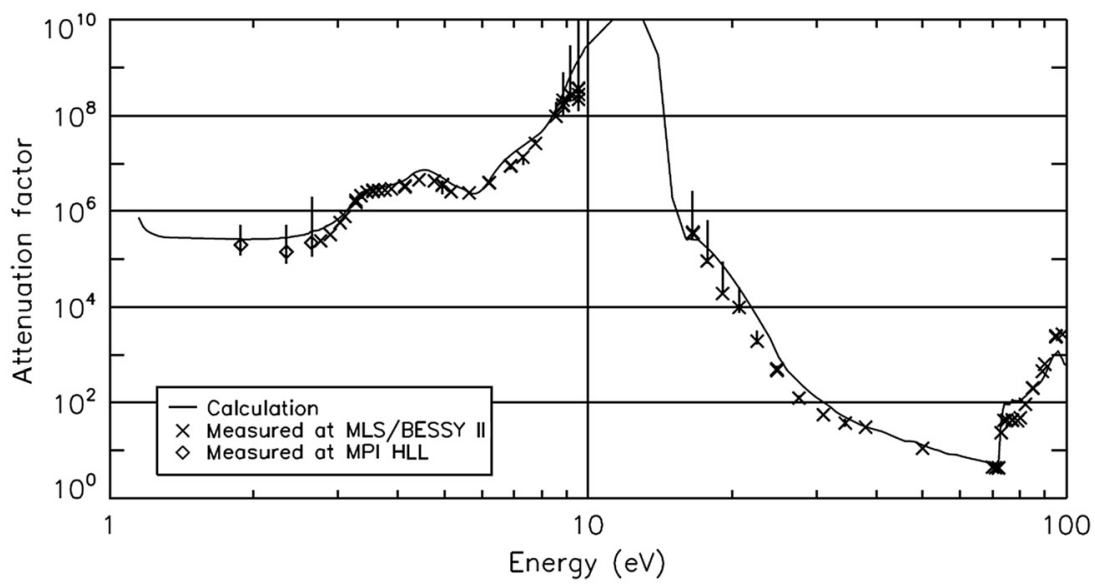

Fig. 11 Attenuation factor of the on-chip filter of TM1 to TM4 and TM6 in the optical und UV band. ${ }^{6}$ The solid line represents a calculation including photon absorption and taking into account the thin film structure of the aluminum layer. The symbols represent the measurement results at MLS/ BESSY II synchrotron facility and at the MPI.

\subsection{Camera Calibration on Ground}

The energy calibration of the eROSITA camera assemblies was performed at the PUMA x-ray test facility of MPE from December 2015 to June 2016. By use of a multi-target x-ray tube, the entire energy range of interest could be calibrated. The results are listed in Tables 4 and 5. Further details of the calibration can be found in Ref. 13.

Only TM7 showed one single bad pixel during ground calibration, whereas all other six cameras were free of any bad pixels. The CTI values (CTI $=1-\mathrm{CTE})$ of the seven detectors varied between $0.6 \times 10^{-5}$ and $3 \times 10^{-5}$ at an energy of $6 \mathrm{keV}$.

\section{Operation in Space}

The commissioning of the seven eROSITA cameras after launch was successfully conducted as planned. However, a few unexpected features were observed. First, the detector operating temperature was higher than planned. Second, the performance of two cameras without on-chip light blocking filter was found to be affected by a light leak in the cover of the focal plane and finally,

Table 4 Energy resolution (FWHM) with one sigma error for all valid events (single, double, triple, and quadruple patterns) of the seven camera assemblies measured in the ground calibration campaign.

\begin{tabular}{|c|c|c|c|c|c|c|c|}
\hline $\begin{array}{l}\text { Spectral line } \\
\text { energy }\end{array}$ & $\begin{array}{c}\text { TM1 } \\
\text { FWHM } \\
(\mathrm{eV})\end{array}$ & $\begin{array}{c}\text { TM2 } \\
\text { FWHM } \\
(\mathrm{eV})\end{array}$ & $\begin{array}{c}\text { TM3 } \\
\text { FWHM } \\
(\mathrm{eV})\end{array}$ & $\begin{array}{c}\text { TM4 } \\
\text { FWHM } \\
(\mathrm{eV})\end{array}$ & $\begin{array}{c}\text { TM5 } \\
\text { FWHM } \\
(\mathrm{eV})\end{array}$ & $\begin{array}{c}\text { TM6 } \\
\text { FWHM } \\
(\mathrm{eV})\end{array}$ & $\begin{array}{c}\text { TM7 } \\
\text { FWHM } \\
(\mathrm{eV})\end{array}$ \\
\hline C-K: 277 eV & $58.2 \pm 0.3$ & $57.8 \pm 0.3$ & $57.7 \pm 0.4$ & $57.8 \pm 0.3$ & $49.7 \pm 0.2$ & $57.6 \pm 0.4$ & $49.3 \pm 0.2$ \\
\hline O-K: $525 \mathrm{eV}$ & $63.9 \pm 0.2$ & $65.2 \pm 0.3$ & $65.7 \pm 0.3$ & $63.5 \pm 0.2$ & $56.7 \pm 0.3$ & $63.4 \pm 0.2$ & $56.3 \pm 0.4$ \\
\hline Cu-L: 930 eV & $70.0 \pm 0.3$ & $74.0 \pm 0.3$ & $72.3 \pm 0.3$ & $69.8 \pm 0.3$ & $67.7 \pm 0.3$ & $69.7 \pm 0.3$ & $68.4 \pm 0.3$ \\
\hline Al-K: $1.49 \mathrm{keV}$ & $77.4 \pm 0.3$ & $81.8 \pm 0.3$ & $80.0 \pm 0.3$ & $77.0 \pm 0.3$ & $75.4 \pm 0.3$ & $77.1 \pm 0.3$ & $77.6 \pm 0.2$ \\
\hline Ti-K $\alpha: 4.51 \mathrm{keV}$ & $118.2 \pm 0.5$ & $125.1 \pm 0.6$ & $122.1 \pm 0.6$ & $118.2 \pm 0.6$ & $115.6 \pm 0.6$ & $118.2 \pm 0.6$ & $116.9 \pm 0.6$ \\
\hline $\mathrm{Fe}-\mathrm{K} \alpha: 6.40 \mathrm{keV}$ & $137.9 \pm 0.6$ & $145.4 \pm 0.7$ & $142.0 \pm 0.7$ & $137.8 \pm 0.6$ & $134.9 \pm 0.7$ & $138.1 \pm 0.7$ & $136.0 \pm 0.7$ \\
\hline $\mathrm{Cu}-\mathrm{K} \alpha: 8.04 \mathrm{keV}$ & $158.3 \pm 0.7$ & $166.6 \pm 0.7$ & $163.1 \pm 0.7$ & $158.5 \pm 0.7$ & $155.1 \pm 0.6$ & $158.5 \pm 0.6$ & $156.2 \pm 0.7$ \\
\hline Ge-K $\alpha: 9.89 \mathrm{keV}$ & $177.5 \pm 1.0$ & $181.0 \pm 1.0$ & $182.0 \pm 1.1$ & $173.4 \pm 1.1$ & $170.3 \pm 1.0$ & $173.6 \pm 1.1$ & $174.5 \pm 1.0$ \\
\hline
\end{tabular}


Table 5 Energy resolution including one sigma error obtained for single events. This event type is used for gain and CTE determination.

\begin{tabular}{|c|c|c|c|c|c|c|c|}
\hline $\begin{array}{l}\text { Spectral line } \\
\text { energy }\end{array}$ & $\begin{array}{c}\text { TM1 } \\
\text { FWHM } \\
(\mathrm{eV})\end{array}$ & $\begin{array}{c}\text { TM2 } \\
\text { FWHM } \\
(\mathrm{eV})\end{array}$ & $\begin{array}{c}\text { TM3 } \\
\text { FWHM } \\
(\mathrm{eV})\end{array}$ & $\begin{array}{c}\text { TM4 } \\
\text { FWHM } \\
(\mathrm{eV})\end{array}$ & $\begin{array}{c}\text { TM5 } \\
\text { FWHM } \\
(\mathrm{eV})\end{array}$ & $\begin{array}{c}\text { TM6 } \\
\text { FWHM } \\
(\mathrm{eV})\end{array}$ & $\begin{array}{c}\text { TM7 } \\
\text { FWHM } \\
(\mathrm{eV})\end{array}$ \\
\hline C-K: 277 eV & $7.2 \pm$ & & & & & & 48.2 \\
\hline O-K: 52 & $1.4 \pm 0.3$ & $62.6 \pm 0.5$ & $62.9 \pm 0.4$ & $61.2 \pm 0.3$ & $54.5 \pm 0.4$ & $61.6 \pm 0.3$ & $53.9 \pm 0.5$ \\
\hline Cu-L: 9 & $66.3 \pm 0$ & $69.4 \pm 0.5$ & $68.0 \pm 0.5$ & $66.1 \pm 0.5$ & $64.6 \pm 0.5$ & $66.1 \pm 0.5$ & $65.3 \pm 0.5$ \\
\hline AI-K: 1.4 & $73.5 \pm 0$ & $76.7 \pm 0.5$ & $75.2 \pm 0.5$ & $73.2 \pm 0.5$ & $72.1 \pm 0.5$ & $73.4 \pm 0.5$ & $73.0 \pm 0.4$ \\
\hline Ti-K $\alpha: 4.51 \mathrm{keV}$ & $113.6 \pm 1.2$ & $117.5 \pm 1.4$ & $115.7 \pm 1.4$ & $113.7 \pm 1.3$ & $111.9 \pm 1.4$ & $113.6 \pm 1.3$ & $113.1 \pm 1.3$ \\
\hline $\mathrm{Fe}-\mathrm{K} \alpha: 6.40 \mathrm{keV}$ & $133.0 \pm 1.6$ & $136.6 \pm 1.9$ & $134.4 \pm 1.8$ & $132.5 \pm 1.5$ & $130.6 \pm 1.7$ & $133.0 \pm 1.8$ & $132.0 \pm 1$ \\
\hline $\mathrm{Cu}-\mathrm{K} \alpha: 8.04 \mathrm{keV}$ & $152.6 \pm 1.8$ & $156.3 \pm 1.8$ & $154.2 \pm 1.8$ & $152.0 \pm 1.7$ & $149.6 \pm 1.6$ & $152.4 \pm 1.6$ & $151.6 \pm 1$ \\
\hline $\mathrm{Ge}-\mathrm{K} \alpha: 9.89 \mathrm{keV}$ & $170.8 \pm 2.5$ & $169.6 \pm 2.8$ & $172.6 \pm 2.9$ & $166.1 \pm 3.0$ & $164.9 \pm 2.8$ & $166.8 \pm 3.1$ & $169.6 \pm 2.6$ \\
\hline
\end{tabular}

the seven CE suffer approximately once a week from SEUs. The latter effect seems to be caused by particles, which deposit energy in the FPGA and generate disturbances in the logic. The TMR avoids SEUs in the FPGA, but the integrated circuit resources were not sufficient to implement the complex firmware and software completely in TMR technique. As a result, resets of the CE are necessary when an SEU occurs and permanently disturbs the camera operation or the data acquisition. After the reset, the operation can be resumed. The detector operating voltages are maintained during the $\mathrm{CE}$ reset and thus no switch off and switch on of the detector is necessary.

The planned operating temperature of $-95^{\circ} \mathrm{C}$ for the CCD sensor was not achieved in space due to a problem in the thermal system and thus the detectors have to be operated at a higher temperature of about $-85^{\circ} \mathrm{C}$. This means that the degradation of energy resolution due to radiation damage will be more significant than expected according to Table 2. However, primarily high x-ray energies are affected, while the degradation is small for low energies.

The lower event thresholds were adjusted in space during the commissioning and calibrationperformance verification phases to match the resulting event rate with the permitted telemetry rate from spacecraft to ground stations. The event threshold is $\sim 75 \mathrm{eV}$ with slight differences for the five cameras with on-chip filter. The vicinity threshold for the neighboring pixels in the four main directions is about $45 \mathrm{eV}$, which allows to detect low-energy split event fractions of an x-ray photon event. This is a major improvement compared to the EPIC-PN camera, which has an event threshold $\geq 100 \mathrm{eV}$. Only camera TM5 and TM7 required a higher event threshold because of the higher noise due to the light leak (see Sec. 5.1).

\subsection{Light Leak}

During commission of the cameras TM5 and TM7, a relative high and inhomogeneous noise over the detector area was observed (see Fig. 12). Since this effect was present with the filter wheel in closed position, the noise changes with the Sun angle, and only the cameras with no on-chip filter were affected. It was obvious that a light leak causes the noise increase. This means that light from the Sun enters the camera array volume, although a cover wrapped with multilayer insulation foil should have shielded it.

A non-uniform light intensity reaches the CCD on the side toward the mirror. The optical photons enter the unshielded and for this wavelength range quite transparent photon entrance window of the CCD sensor surface and generate a number of electrons proportional to the incident light intensity. Since the light is non-uniformly distributed over the CCD area, the common mode correction, which subtracts the median of a 64-pixels large block of a row, is corrupted. This effect and the noise contribution caused by the optical photons, necessitate a higher event threshold to distinguish photon signals from noise. By setting a higher primary event threshold of about $125 \mathrm{eV}$ for TM5 and $\sim 145 \mathrm{eV}$ for TM7, observations can be performed in spite of the light 


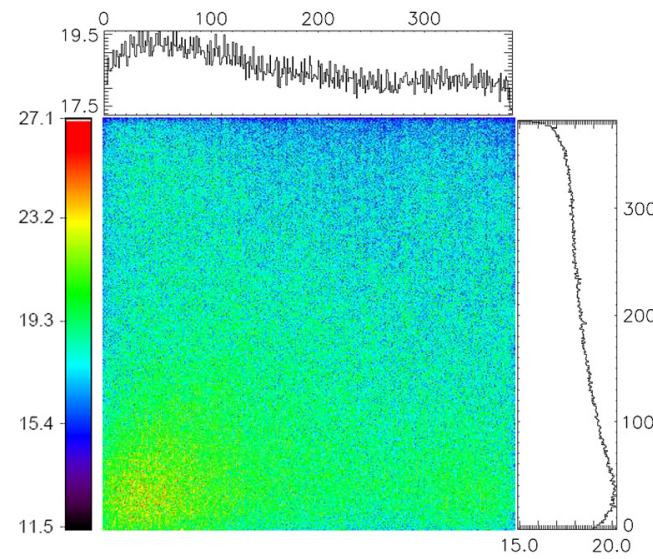

(a)

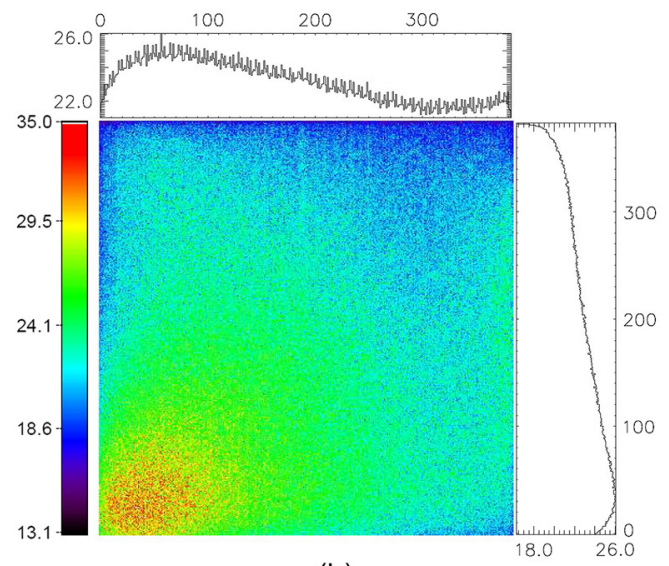

(b)

Fig. 12 Noise maps of (a) TM5 and (b) TM7 taken during the commission phase showing the increased and non-uniform noise over the image consisting of $384 \times 384$ pixels. The typical noise value of the other cameras is $\sim 13$ adu.

leak effects. During the first all-sky-survey, the dependence of the light intensity on the orientation of the telescope with respect to the Sun could be studied and a clear correlation was found (see Ref. 1, Fig. 18). This offers the possibility to develop specific calibration algorithms, which take the orientation into account.

\subsection{Energy Resolution}

The energy resolutions of the seven cameras were determined in space by use of the onboard calibration sources (see Table 6). They are approximately the same as measured on ground, see Table 5.

\subsection{First Light}

After the successful commissioning phase, all seven eROSITA TMs observe the x-ray sky since October 15, 2019. Figure 13 shows the first light of the eROSITA x-ray telescope pointing to a field in the LMC. The image shows impressively the capability of the eROSITA instrument and in particular of the innovative cameras.

\subsection{Micrometeoroid Damage}

On December 5, 2019 around 21:30 Moscow time, a sudden increase of the light curve was observed for camera TM4 (see Fig. 14). Analysis showed that this effect was caused by four

Table 6 Energy resolution including the one sigma error of the seven eROSITA cameras in space. ${ }^{13}$ The values were obtained from onboard calibration source measurements in November and December 2019. The operating temperature was $-85^{\circ} \mathrm{C}$. The gain and CTE determination is done with single events. For this reason, the energy resolution is shown for single events in this table. The values of TM5 and TM7 vary more than those of the other TMs because they depend on the Sun angle due to the light leak effect.

\begin{tabular}{|c|c|c|c|c|c|c|c|}
\hline $\begin{array}{l}\text { Spectral line } \\
\text { energy }\end{array}$ & $\begin{array}{c}\text { TM1 } \\
\text { FWHM } \\
(\mathrm{eV})\end{array}$ & $\begin{array}{c}\text { TM2 } \\
\text { FWHM } \\
(\mathrm{eV})\end{array}$ & $\begin{array}{c}\text { TM3 } \\
\text { FWHM } \\
(\mathrm{eV})\end{array}$ & $\begin{array}{c}\text { TM4 } \\
\text { FWHM } \\
(\mathrm{eV})\end{array}$ & $\begin{array}{c}\text { TM5 } \\
\text { FWHM } \\
(\mathrm{eV})\end{array}$ & $\begin{array}{c}\text { TM6 } \\
\text { FWHM } \\
(\mathrm{eV})\end{array}$ & $\begin{array}{l}\text { TM7 } \\
\text { FWHM } \\
(\mathrm{eV})\end{array}$ \\
\hline Al-K: $1.49 \mathrm{keV}$ & $74.4 \pm 0.7$ & $78.3 \pm 0.7$ & $75.9 \pm 0.9$ & $73.9 \pm 0.8$ & $79.7 \pm 1.2$ & $73.4 \pm 0.7$ & $90.2 \pm 1.2$ \\
\hline Ti-K $\alpha: 4.51 \mathrm{keV}$ & $116.5 \pm 0.4$ & $120.3 \pm 0.5$ & $118.3 \pm 0.5$ & $115.9 \pm 0.4$ & $118.1 \pm 0.6$ & $115.2 \pm 0.4$ & $128.0 \pm 0.6$ \\
\hline $\mathrm{Mn}-\mathrm{K} \alpha: 5.89 \mathrm{keV}$ & $136.8 \pm 1.9$ & $141.6 \pm 2.5$ & $137.6 \pm 3.0$ & $136.5 \pm 2.7$ & $137.4 \pm 3.2$ & $136.6 \pm 2.1$ & $145.9 \pm 3.1$ \\
\hline
\end{tabular}




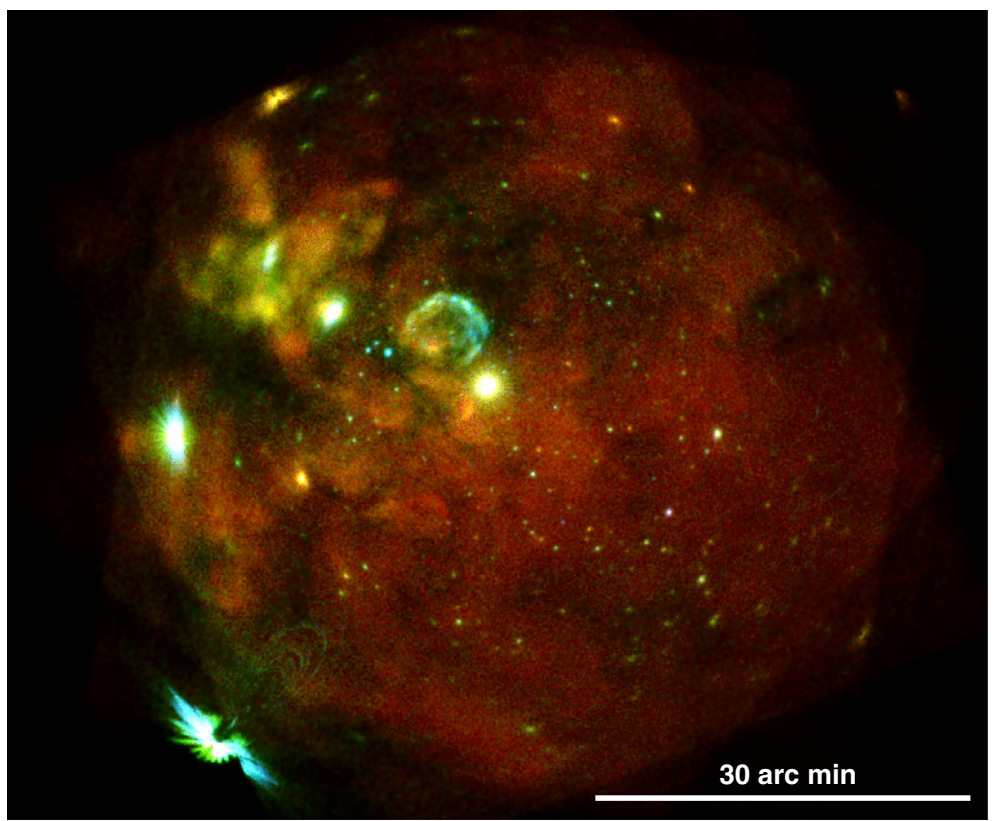

Fig. 13 First light of the eROSITA x-ray telescope in space. ${ }^{1}$ A field in the LMC was chosen as target, with the pointing centered on the supernova SN 1987A. Red, green, and blue colors represent $\mathrm{x}$-ray intensities in the 0.2 to $1.0,1.0$ to 2.0 , and 2.0 to $4.5 \mathrm{keV}$ energy bands.

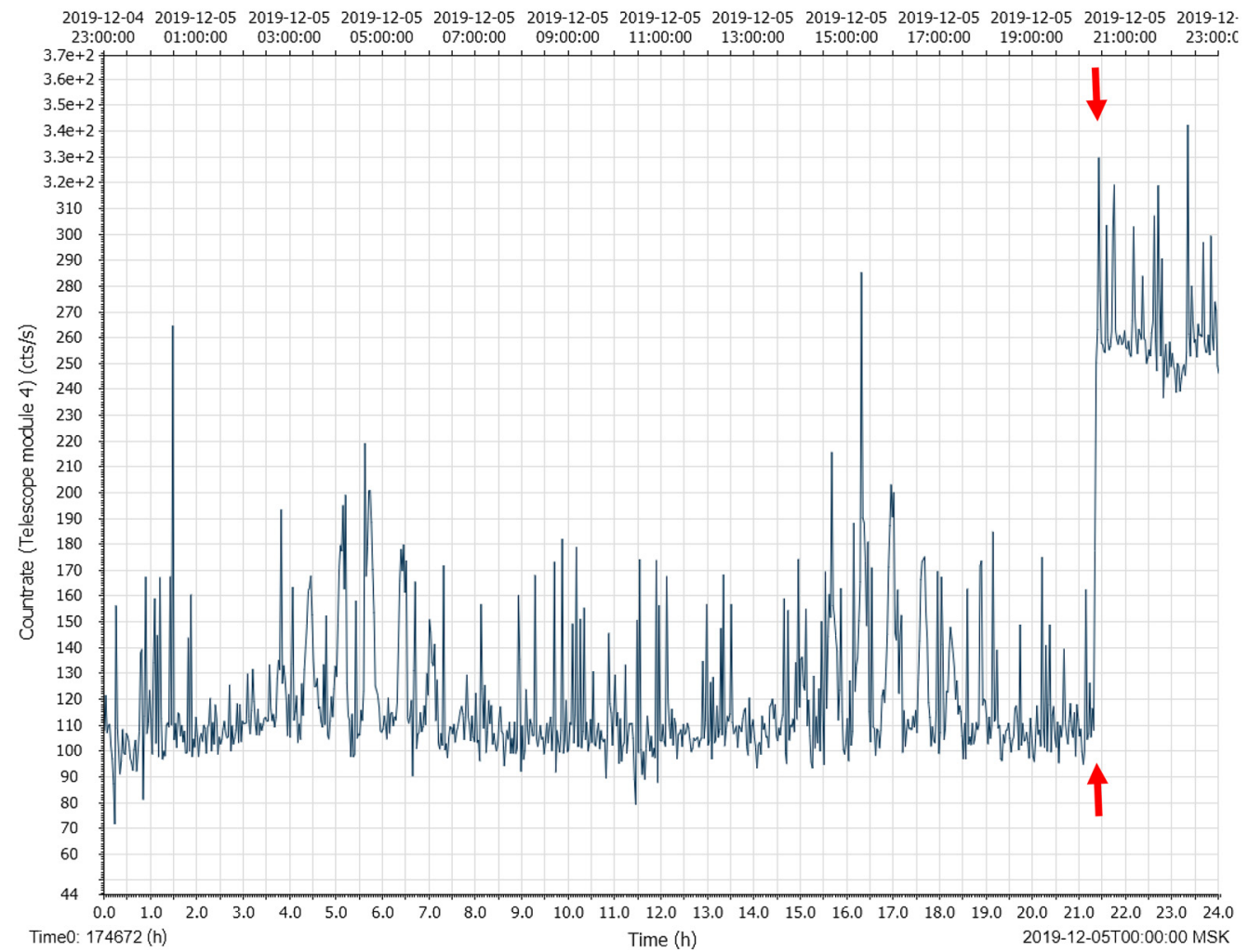

Fig. 14 Sudden and permanent count rate increase of TM4, around 21:30 on December 5, 2019, Moscow time.

pixels in a square, located in channels 302 and 303 and rows 82 and 83, which appear since that time permanently bright. Their increase of the offset and noise values clearly indicate a small micrometeoroid hit with an associated damage of the silicon lattice. Such an event was already observed with EPIC-PN on XMM-Newton in 2000 but to a much more severe extent. 


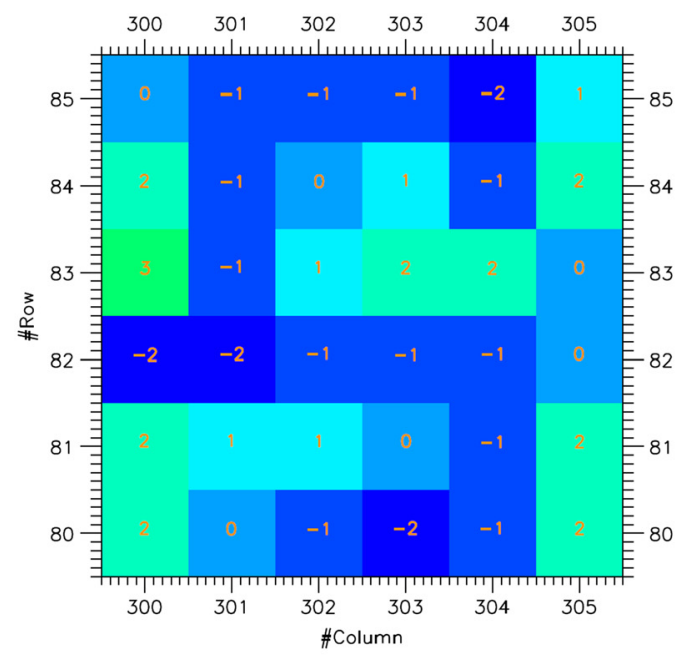

(a)

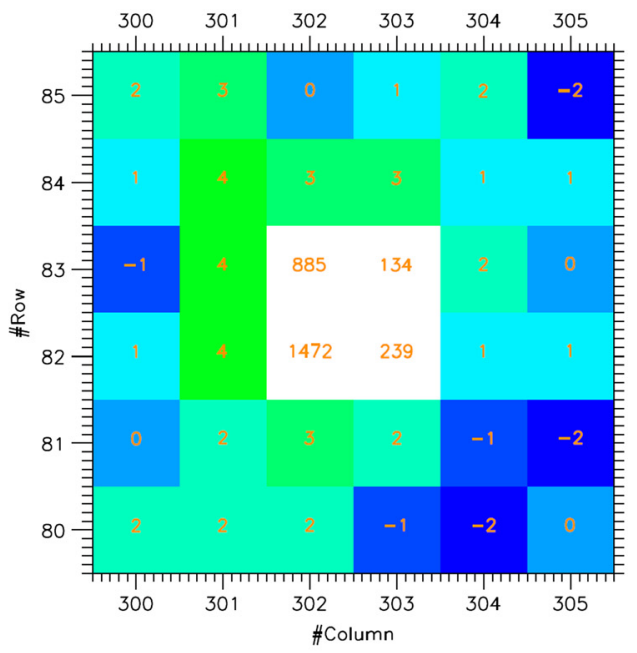

(b)

Fig. 15 Change of pixel offset after micrometeoroid impact in the pixels of columns 302 and 303 and rows 82 and 83 . The offset values (a) before impact and (b) after impact.

About 35 pixels were damaged and their dark current increase was larger. ${ }^{14}$ We assume that in case of eROSITA TM4, the micrometeoroid size was smaller or its speed was lower compared to that on EPIC-PN. We should also note that the 200-nm-thick aluminum layer of the on-chip filter has likely also mitigated the defect in the silicon lattice.

The change of the offset values as shown in Fig. 15 reveals clearly the damage. Above $1 \mathrm{keV}$ one adu corresponds to about $0.76 \mathrm{eV}$. The most severely damaged pixel $(302,82)$ has an increase of about 1470 adu or $1.12 \mathrm{keV}$ corresponding to about 300 electrons, which are continuously generated during $50 \mathrm{~ms}$ in this pixel. The lattice damage and resulting dark current increase cause a noise increase of the four pixels. We observe a noise increase by a factor of 2 for the least affected pixel and by a factor of 5 for the most damaged pixel. While the offset change can be corrected for observations by calculation of a new offset map, the associated noise contribution cannot be eliminated. It causes a degradation of the energy resolution. The performance of the other pixels of the CCD is not affected, not even that of the pixels in the two affected transfer channels.

\section{Summary and Outlook}

The detectors for eROSITA have been developed based on the EPIC-PN detector but taking into account lessons learned during the XMM-Newton project. Several improvements were implemented in the innovative eROSITA detectors by a variety of optimizations. Important examples are the improved low-energy response, almost complete elimination of out-of-time event occurrence, substantially smaller charge transfer losses, significantly lower read noise and higher energy resolution. In addition, a higher robustness with respect to environmental conditions was achieved by passivation of the front surface and an optical light blocking filter directly deposited on the sensor.

A peculiarity of the seven eROSITA camera assemblies is that they are not designed as a vacuum enclosure for the susceptible ultra-thin optical blocking filter and detector. Both are exposed to the acoustic noise arising during satellite launch and are not protected against contamination during this phase.

In the course of the development, an engineering model, a QM, and finally the seven flight models have been assembled. Performance tests and environmental tests were conducted to verify the detector and camera design, completed by calibration of the camera assemblies.

The wide field, high-throughput eROSITA x-ray telescope was launched in July 2019 and orbits around the second Lagrange point of the Sun-Earth system. The seven CCD cameras with the innovative PNCCD detectors allow for observations in the energy range of 0.2 to $8 \mathrm{keV}$ with 
high QE and excellent energy, spatial, and time resolution. After successful commissioning in space including calibration and performance verification, the all-sky survey started in December 2019. Some conditions for the camera operation were different that had been expected, such as the occasional occurrence of SEUs in the CE, insufficient shielding of optical light for the focal plane area, and a by about $10 \mathrm{~K}$ higher detector operating temperature. Other incidents, such as micrometeoroid impacts have been anticipated as already known from other X-ray observatories like XMM-Newton. Nonetheless, the effects are relatively small or can be mitigated so that the eROSITA $x$-ray telescope has best prerequisites to achieve its scientific goals. This is largely based on the performance of the eROSITA cameras, which have demonstrated in space a quality comparable to that measured on ground.

\section{Acknowledgments}

The authors would like to thank the whole eROSITA project team and all colleagues, who contributed to the development or tests from the Max-Planck-Institut für extraterrestrische Physik, the Hybridlabor of the Max-Planck-Institut für Physik, MPI HLL, in particular Danilo Mießner, and all others taking part in the project. This work was supported by Max-Planck-Gesellschaft and Deutsches Zentrum für Luft- und Raumfahrt. A previous version of this manuscript had been submitted to Proc. of SPIE, Vol. 11444 (2020).

\section{References}

1. P. Predehl et al., "The eROSITA X-ray telescope on SRG," Astron. Astrophys. 647, A1 (2021).

2. N. Meidinger et al., "Report on the eROSITA camera system," Proc. SPIE 9144, 91441W (2014).

3. L. Strüder et al., "The European photon imaging camera on XMM-Newton: the PN-CCD camera," Astron. Astrophys. 365(1), L18-L26 (2001).

4. S. Herrmann et al., "Mixed signal PNCCD readout ASIC for the future X-ray astronomy mission eROSITA," in IEEE Nucl. Sci. Symp. Conf. Rec., Vol. N54-3, pp. 2398-2403 (2007).

5. N. Meidinger et al., "Status of the CCD camera for the eROSITA space telescope," Proc. SPIE 8145, 814502 (2011).

6. S. Granato, "The response of PNCCDs with aluminum on-chip filter to visible, UV- and X-ray radiation," $\mathrm{PhD}$ thesis, https://dspace.ub.uni-siegen.de/handle/ubsi/655 (2012).

7. N. Meidinger et al., "First measurements with a frame store PN-CCD X-ray detector," Nucl. Instrum. Methods A 512, 341-349 (2003).

8. N. Meidinger et al., "Design and performance of the eROSITA focal plane instrumentation," Proc. SPIE 8453, 84530P (2012).

9. N. Meidinger et al., "Progress of the X-ray CCD camera development for the eROSITA telescope," Proc. SPIE 8859, 88590B (2013).

10. N. Meidinger et al., "Development of the focal plane PNCCD camera system for the X-ray space telescope eROSITA," Nucl. Instrum. Methods A 624, 321-329 (2010).

11. D. Coutinho et al., "eROSITA system functionality and operation," Proc. SPIE 10699, 106995F (2018).

12. N. Meidinger et al., "CCD detector development for the eROSITA space telescope," in IEEE Nuclear Sci. Symp. Conf. Rec., Vol. N02-1, pp. 24-31 (2010).

13. K. Dennerl et al., "The calibration of eROSITA on SRG," Proc. SPIE 11444, 114444Q (2020).

14. L. Strüder, et al, "Evidence for micrometeoroid damage in the pn-CCDcamera system aboard XMM-Newton," Astron. Astrophys. 375, L5-L8 (2001).

Norbert Meidinger is a senior staff instrument scientist and project manager at Max-PlanckInstitut fuer extraterrestrische Physik (MPE), Garching, Germany. His research areas include the development of spectroscopic x-ray detectors and instrumentation for satellites. He was 
engaged in the development of PNCCD detector and EPIC-PN camera for ESA's space telescope XMM-Newton. He was responsible for the eROSITA camera development with advanced PNCCD detectors and is senior CoI of eROSITA space telescope. Since the launch in 2019, he has been a member of the eROSITA operations team. Since 2013, he is the project manager of the ATHENA Wide Field Imager (WFI).

Tanja Eraerds (nee Rommerskirchen) received her $\mathrm{PhD}$ in physics from Zurich University in October 2010. Since 2011, she has been working as a research assistant at MPE, where she contributed to the development of the eROSITA x-ray satellite instrument and to the ATHENA WFI. Her interests include data analysis, the usage of Monte Carlo simulations for design optimizations, and the application of DEPFET detectors for space telescopes.

Olaf Hälker is an electronic engineer at MPE, Garching, Germany. His research areas include the development of spectroscopic x-ray detectors particularly camera head electronics for satellites. He was a developer of PNCCD camera head electronics for x-ray free electron lasers and was engaged in design and test of the eROSITA CCD detector flight electronics. Since 2014, he is a developer of ATHENA WFI camera head electronics.

Jonas Reiffers is an electronics developer and electronics team coordinator at MPE, Garching, Germany. He engaged in the development and test of space qualified (ESA) flight electronics for the detector electronics of ESA's ATHENA WFI. His areas of interests include the development and support of FPGA-based laboratory test environments for the test and characterization of $\mathrm{x}$-ray detector systems and Readout ASICs for the eROSITA Satellite Mission and for Mercury Imaging X-ray Spectrometer as part of BepiColombo. Formerly he worked on EMC investigations of bus systems in the automotive sector.

Biographies of the other authors are not available. 